\title{
Bandwidth-limited few-cycle pulses by nonlinear compression in a dispersion-alternating fiber
}

\author{
Niklas M. Lüpken ${ }^{1}$. Carsten Fallnich ${ }^{1,2}$
}

Received: 15 June 2020 / Accepted: 8 October 2020 / Published online: 20 October 2020

(c) The Author(s) 2020

\begin{abstract}
We demonstrate an improved concept for nearly bandwidth-limited nonlinear pulse compression down to the few-cycle regime in a fiber chain with alternating sign of dispersion. Whereas the normally dispersive fiber segments generate bandwidth via self-phase modulation, the anomalously dispersive fiber segments recompress the broadened spectral bandwidth by an appropriate amount of group velocity dispersion. Nonlinear pulse compression from 80 fs input pulses to nearly bandwidth-limited $25 \mathrm{fs}$ pulses at $1560 \mathrm{~nm}$ was achieved, resulting in a pulse compression factor of 3.2. The use of a specific dispersion-compensating fiber eliminated the impact of higher-order dispersion, such that a high spectral coherence was ensured. We show that nonlinear Schrödinger equation simulations were in good agreement with the experimental results and investigated the transfer of input fluctuations to the output. The concept is transferable to longer input pulse durations, resulting in compression factors of 83 for 10 ps input pulses.
\end{abstract}

\section{Introduction}

Ultra-short pulses deliver high peak power within a very short time interval; therefore, pulse compression is a topic of high interest for a manifold of applications such as highharmonic generation [1-3], supercontinuum generation [4, 5], nonlinear optical microscopy [6], and telecommunication [7]. To generate ultra-short optical pulses, a broad bandwidth is necessary. For instance, gain media like titanium:sapphire crystals offer a broad bandwidth for the generation of pulses with durations down to a few femtoseconds [8]. Gain media doped with chromium ions provide enough bandwidth for the generation of ultra-short pulses in the telecom wavelength range [9], e.g., for high-speed optical time-division multiplexing [7]. However, fiber-based gain media are preferred in many applications such as telecommunications or biological imaging, but do not offer such a broad bandwidth. Due to the limited bandwidth of typical laser gain media such as erbium or ytterbium glass, pulse durations in the order of many tens or even hundreds of femtoseconds are

Niklas M. Lüpken

n.luepken@uni-muenster.de

1 Institute of Applied Physics, University of Münster, Corrensstraß e 2, 48149 Münster, Germany

2 MESA+ Institute for Nanotechnology, University of Twente, 7500 AE Enschede, The Netherlands extracted directly from oscillators but the few-cycle regime is hardly accessible.

To overcome this problem, nonlinear pulse compression (NLPC) has been well established [10]. This technique utilizes spectral broadening in a Kerr medium with subsequent compensation of the induced frequency chirp. NLPC was achieved within many different schemes using photonic crystal fibers [2, 11-14], highly nonlinear fibers [15], large-mode-area fibers [1, 3], hollow-core fibers [16-18], fiber amplifiers [19, 20], or even multi-pass cells [21]. With this NLPC concept, high pulse compression factors up to 19 were achieved routinely [2]; however, very high pulse energies such as $0.5 \mathrm{~mJ}$ [17] were required to generate the necessary bandwidth. Nevertheless, NLPC for the generation of few-cycle pulses was also demonstrated with input pulse energies in the nanojoule range [22-25]. However, to compress the achieved bandwidth to ultra-short pulse durations, higher-order dispersion (HOD) has to be considered and all these systems require external compressors, which have to be aligned.

In contrast, soliton-effect pulse compression utilizes soliton formation to shorten the pulses without any type of external compression. This technique relies on temporal focusing by self-phase modulation in conjunction with anomalous dispersion in a nonlinear waveguiding medium, and ultra-short pulses can be extracted close to the soliton fission point [26]. Soliton-effect pulse compression was 
demonstrated in various waveguides such as optical fibers [27-29], photonic nanowires [30], photonic crystal fibers [31-33], gas-filled Kagome fibers [18, 34], photonic crystal waveguides [35], and silicon nitride waveguides [4]. Compression factors of 18 have been demonstrated [34], and low-power pulse compression with nanojoule-level or even picojoule-level pulse energies is possible [32, 33, 36]. However, to form a soliton, the input pulse energy has to be equal to or larger than the soliton energy, and the output pulse duration is very sensitive to input energy fluctuations or noise [37]. Furthermore, the compression factor scales with the soliton number and, therefore, with the input peak power [38]. Additionally, the pulse quality is limited at the point of maximum compression as part of the energy is contained in a temporally broad pedestal [39]. Nevertheless, for a soliton order of 5 roughly $70 \%$ of the pulse energy are contained in the compressed pulse [27]. However, pulse qualities reaching up to $90 \%$ of the initial pulse energy in the compressed pulse are hardly achieved.

Dispersion-alternating fibers (DAF) combine the advantages of ordinary pulse compression and soliton-effect pulse compression and, therewith, overcome typical limitations for bandwidth generation in optical fibers, such as dispersive temporal defocusing and soliton formation [5, 7, 40, 41]. The alternation of the dispersion sign allows for repetitive sections of SPM-mediated bandwidth generation in the normal dispersion regime with subsequent pulse compression in the anomalous dispersion regime. Then, NLPC can be achieved without the use of free-space compressors or soliton formation, and a high compression factor of about 19 to approximately $380 \mathrm{fs}$ pulse duration was achieved [7]. Nonetheless, due to the non-inverted dispersion slopes of the involved fibers, the output pulses suffered from HOD leading to wave-breaking $[5,7]$. Wave-breaking prohibits the recompression to the bandwidth-limited pulse duration with grating or prism compressors, compensating typically only second-order dispersion.

Here, we present a DAF consisting of anomalously and normally dispersive fibers with matched dispersion profiles in terms of the dispersion magnitude and slope to generate bandwidth-limited few-cycle pulses. To achieve bandwidthlimited pulses, HOD impact has to be minimized during pulse compression by matching the dispersion magnitude $D$ and dispersion slope $S$ of the involved fibers:

$\frac{D_{\text {normal }}}{D_{\text {anomalous }}}=\frac{S_{\text {normal }}}{S_{\text {anomalous }}}=-R$,

where $R$ corresponds to the dispersion ratio. In previous experiments, the dispersion magnitude was matched, but the dispersion slope of both fibers was positive, leading to an increased impact of HOD [5]. In our experiments, avoidance of HOD was accomplished by utilizing standard SMF28 fiber in combination with a specific dispersion-compensating fiber (DCF, Corning Inc.). According to the data of the manufacturers, the ratio of the dispersion magnitude and dispersion slope at $1550 \mathrm{~nm}$ wavelength of both types of fiber corresponds to

$\frac{D_{\mathrm{DCF}}}{D_{\mathrm{SMF} 28}}=\frac{S_{\mathrm{DCF}}}{S_{\mathrm{SMF} 28}} \approx-6.7$.

This ratio is fixed by the design of the DCF and does not influence the compression ratio but corresponds to the necessary length ratio of the two different types of fibers to compensate the overall dispersion (in the case of linear propagation). With input pulse energies of $1.3 \mathrm{~nJ}$ pulse compression from initially $80 \mathrm{fs}$ down to $25 \mathrm{fs}$, towards the few-cycle regime, was achieved. We show that this concept can be transferred to longer input pulse durations, achieving compression ratios of up to 83 for 10 ps input pulses.

\section{Numerical methods}

The experiments presented in the following were supported by numerical simulations of the generalized scalar nonlinear Schrödinger equation [42]:

$\frac{\partial A}{\partial z}=\sum_{k \geq 2} \frac{i^{k+1}}{k !} \beta_{k} \frac{\partial^{k} A}{\partial t^{k}}+i \gamma\left(1+i \tau_{\text {shock }} \frac{\partial}{\partial t}\right)|A|^{2} A$,

where $A$ denotes the temporal envelope of the pulse, $z$ the propagation distance, $\beta_{k}$ the $k$-th dispersion coefficient, $t$ the time in the co-moving frame of the pulse envelope, $\gamma$ the nonlinear coefficient, $\tau_{\text {shock }}$ the shock constant, and linear propagation losses and Raman effects were neglected, as they did not alter the simulation results within comparative calculations.

To simulate the nonlinear pulse propagation in the DAF, the dispersion curves of both fibers have to be modeled as a function of the wavelength $\lambda$. The dispersion profile of the $\operatorname{SMF} 28$ fiber $\left(D_{\text {SMF28 }}(1550 \mathrm{~nm}) \approx+18 \mathrm{ps} / \mathrm{nm} / \mathrm{km}\right)$ was calculated with a mode solver and is shown as a solid blue curve in Fig. 1. On account of missing information on the refractive index profile and the dispersion profile of the DCF, the dispersion of the DCF was measured by spectral white light interferometry with an Erbium-doped ASE source, such as it was described in Ref. [43]. Within the measurement uncertainties the determined dispersion values (see black line in Fig. 1) match with the assumed dispersion $-120 \mathrm{ps} / \mathrm{nm} / \mathrm{km}$ at $1550 \mathrm{~nm}$ (compare Eq. 2) as well as with the dispersion slope. For the simulations, the dispersion of the DCF was assumed to completely compensate the dispersion of the SMF28 fiber, leading to $D_{\mathrm{DCF}}(\lambda)=-6.7 \cdot D_{\mathrm{SMF} 28}(\lambda)$, see Eq. (2). This assumption was justified by a good agreement between experimental results and numerical simulations. 


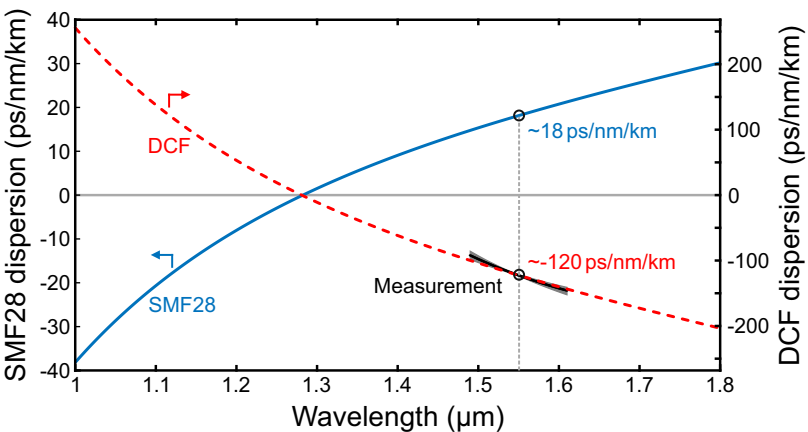

Fig. 1 Calculated dispersion coefficient as a function of the wavelength for the SMF28 fiber (solid blue curve) and the DCF (dashed red curve). The dispersion profile of the DCF was calculated from the SMF28 dispersion with the factor of -6.7 . The black line shows the measured dispersion with the measurement uncertainty as a gray shaded area

The dispersion profile of the DCF is shown in Fig. 1 as a dashed red curve.

\section{Scheme}

The pulse compression in a DAF works as follows: The first normally dispersive fiber segment generates bandwidth via self-phase modulation (SPM), while the pulse is defocusing temporally due to group velocity dispersion, resulting in a reduced peak power, stopping the bandwidth generation eventually. To recompress the pulse, a second piece of fiber with anomalous dispersion is added, and the resulting pulse duration is even shorter than the initial pulse duration due to the additional spectral bandwidth. During this recompression stage more additional bandwidth can be generated by SPM. The length of the anomalously dispersive fiber is chosen such that the temporal focus is reached at its end. The length of this fiber depends on the chirp induced by the preceding normally dispersive segment and on the chirp of the input pulses. Then, a normally dispersive fiber segment is added to generate even more bandwidth via SPM. The resulting bandwidth is then recompressed with a fourth piece of fiber with anomalous dispersion. This process of alternating dispersion can be repeated to subsequently increase bandwidth and to achieve shorter pulse durations than in previous fiber segments by recompression, eventually resulting in ultra-short few-cycle pulses. However, the achievable compression factor in the experiments is limited due to splice losses at the fiber interfaces of the individual segments. Furthermore, HOD does not limit the achievable compression factor as it is minimized by the design of the DAF; however, the zero-dispersion wavelength of the SMF28 fiber near $1.3 \mu \mathrm{m}$ wavelength (see Fig. 1) would limit the achievable pulse compression as the fiber is normally dispersive for shorter wavelengths. Accordingly, the shortest achievable pulse duration would be about 5 fs if a maximal bandwidth of $500 \mathrm{~nm}$ centered at $1550 \mathrm{~nm}$ wavelength is assumed.

Concerning the experimental realization, an optical spectrum analyzer was used to determine the appropriate lengths of the normally dispersive fiber segments to maximize bandwidth generation, and an autocorrelator was used to measure the pulse duration when cutting back the anomalously dispersive fiber segments to the temporal focus, i.e., the point of maximum recompression.

The length and transmission values of each fiber segment in the characterized DAF are denoted in Table 1. The losses in the segments resulted from splicing losses of the two fibers with mode-field diameters of $10.4 \mu \mathrm{m}$ and $4.2 \mu \mathrm{m}$ of the SMF28 and DCF, respectively, measured with a beam profiler (BP104-IR2 from Thorlabs). These losses added up to $-5.34 \mathrm{~dB}$ overall transmission, which could be overcome in future experiments by tapering for improved mode-matching of both fiber types.

\section{Results and discussion}

Figure 2 shows a schematic of the experimental setup. Pulses with a repetition rate of $80 \mathrm{MHz}$ and a duration of $80 \mathrm{fs}$ centered at $1560 \mathrm{~nm}$ originating from a mode-locked Erdoped fiber laser (Er-Laser; FemtoFiber Pro IR from Toptica) were coupled into a DAF, consisting of alternating DCF and SMF28 fiber segments, using an aspheric lens (L1).

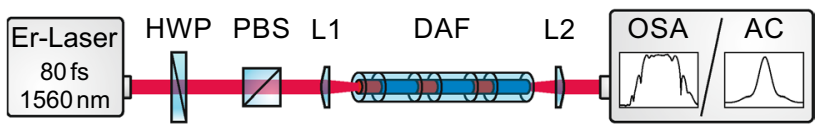

Fig. 2 Schematic of the experimental setup. Pulses from an Er-doped fiber laser were coupled into a dispersion-alternating fiber (DAF). The output pulses were characterized with an optical spectrum analyzer (OSA) and an autocorrelator (AC). $H W P$ half-wave plate, $P B S$ polarizing beam splitter, $L$ aspherical lens. For details see text

Table 1 Length and transmission of each fiber segment of the used dispersion-alternating fiber chain. The transmission values inside the parentheses include the input coupling efficiency

\begin{tabular}{llll}
\hline Segment & Fiber type & Length $(\mathrm{cm})$ & Transmission $(\mathrm{dB})$ \\
\hline 1 & DCF & 4 & $(-1.60)$ \\
2 & SMF28 & 21 & -1.37 \\
3 & DCF & 3 & -0.56 \\
4 & SMF28 & 20 & -1.41 \\
5 & DCF & 3 & -0.60 \\
6 & SMF28 & 20 & -1.40 \\
Total & & 71 & $-5.34(-6.94)$ \\
\hline
\end{tabular}


Least-square fitting autocorrelation functions of Gaussianand $\operatorname{sech}^{2}$-shaped pulses to the measured autocorrelation traces confirmed in accordance to their smallest deviations that the input pulses were $\operatorname{sech}^{2}$-shaped. The input pulse energy could be adjusted with a half-wave plate (HWP) and a polarizing beam splitter (PBS). The fiber segments were spliced together according to the procedure described above (see also Table 1). The output light of the DAF was collected with a second aspheric lens (L2) and then characterized with an optical spectrum analyzer (OSA, AQ-6370D from Yokogawa) and an autocorrelator (AC, pulseCheck from APE).

Figure 3 depicts the spectra and autocorrelation traces of the input and output pulses of the DAF with a launched pulse energy of $1.3 \mathrm{~nJ}$ inside the fiber. Note that we state the input pulse energy as the pulse compression in this scheme is independent of the repetition rate. The spectral full-width at half-maximum (FWHM) bandwidth increased from 71 to $110 \mathrm{~nm}$ (see Fig. 3a) at the output of the DAF. The additionally generated bandwidth accomplished to reduce the pulse duration from 80 fs down to 25 fs as shown by the autocorrelation traces in Fig. 3b, resulting in a pulse compression factor of 3.2. This pulse duration corresponds to less than five optical cycles of the electrical field. The measured pulse duration matched the bandwidth-limited pulse duration of approximately $24 \mathrm{fs}$ (gray shaded area in Fig. 3b) due to the matching dispersion profiles of the used fibers and, therefore, represented the minimized impact of HOD. Only a small amount of energy was located in the wings around \pm 150 fs delay; these features were also observable in the input autocorrelation trace. However, a small portion of the pulse energy could probably be transferred to satellite pulses, being responsible for such features, due to nonlinear chirp contributions induced by SPM, which cannot be compensated by the fiber's group velocity dispersion.

The pulse quality of the output pulses, here defined as the energy fraction contained in the main compressed peak,
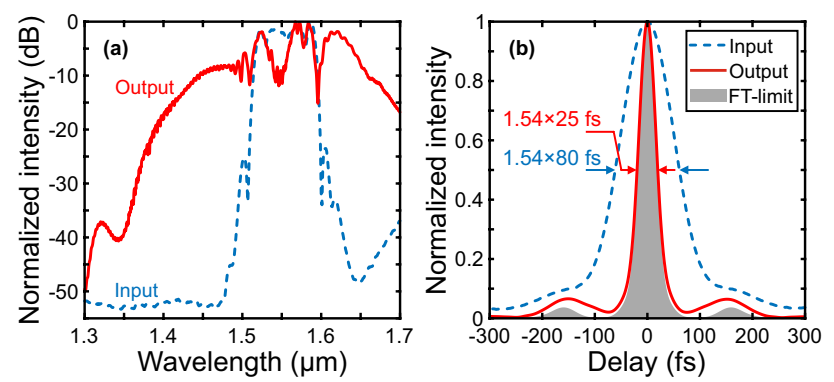

Fig. 3 a Normalized spectrum and $\mathbf{b}$ normalized autocorrelation trace of the pulsed input (dashed blue lines) and output (solid red lines) of the dispersion-alternating fiber. The gray shaded area depicts the bandwidth-limited autocorrelation trace. The numbers denote the full-width at half-maximum pulse duration reached about $87.2 \%$ and was not significantly degraded in comparison to the input pulse quality of roughly $90 \%$. The energy fractions were estimated from the autocorrelation traces shown in Fig. 3b.

The dynamics of the NLPC in the DAF are depicted in Fig. 4, where the simulated pulse duration (solid blue curve) and the simulated $-30 \mathrm{~dB}$ full spectral bandwidth (dashed red curve) are plotted as a function of the propagation distance. Sech ${ }^{2}$-shaped input pulses were launched including a linear down-chirp ( $C=-0.35$; compare with Ref. [26] induced by optical components in the beam path before the input coupling; the induced group delay dispersion was approximately $-770 \mathrm{fs}^{2}$. The pulse duration increased in the normally dispersive fiber segments and was recompressed in the anomalously dispersive fiber segments while the spectral bandwidth increased continuously due to the nonlinear dynamics discussed earlier. In addition to the simulated data the measured pulse durations (blue squares), which were estimated from autocorrelation traces with a deconvolution factor of 1.54 , and the measured spectral bandwidths (red dots) are denoted in Fig. 4. The simulated pulse durations agree very well with the measured pulse durations. The deviations between simulated and experimental data, which increase with the propagation distance, originated from uncertainties of the segment lengths in the DAF and were accumulating with increasing segment number. The simulated and measured spectral bandwidths match after the first two fiber segments but deviate in the subsequent segments. The increase in spectral bandwidth in the third fiber segment is smaller in the simulations compared to the experiment. By our numerical simulations we were able to identify that the main reason for these deviations was the non-perfectly $\operatorname{sech}^{2}$-shaped input spectrum in the experiments (compare Fig. 3a). As the spectral bandwidth was estimated by a $-30 \mathrm{~dB}$ threshold, spectral components apart

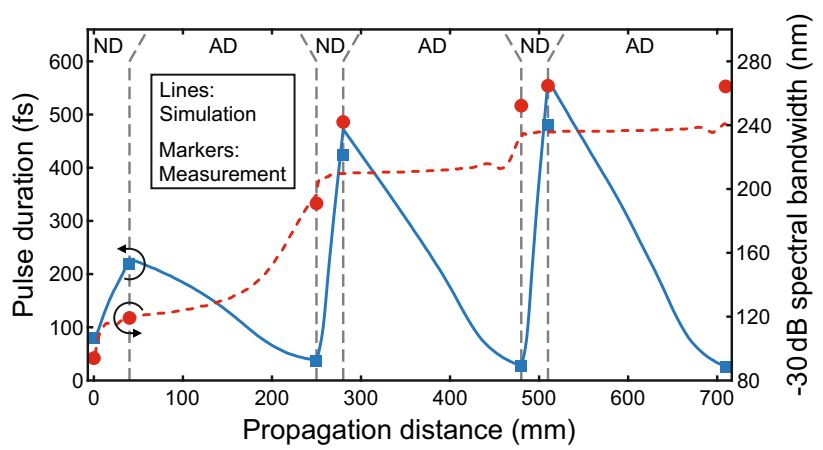

Fig. 4 Evolution of the pulse duration (solid blue curve) and spectral bandwidth (dashed red curve) of the simulated pulse propagation in a dispersion-alternating fiber. The markers correspond to the measured pulse duration (blue squares) and the measured spectral bandwidth (red dots). Vertical dashed lines indicate the change of fiber type with normal and anomalous dispersion (ND and AD) 
from the main spectrum, which increase above the threshold, could have increased the bandwidth estimation. Furthermore, a wavelength-dependent loss occurred on account of the different mode-field diameters of the involved fibers [44], which was not included in the simulations because the wavelength-dependent mode profiles could not be calculated for the DCF on account of missing refractive index profiles. Nevertheless, with the $\operatorname{sech}^{2}$-shaped input spectrum and an averaged wavelength-independent splice loss in the simulations, a good agreement between simulation and experiment concerning the relative changes of the spectral bandwidth in the last three fiber segments was observed.

The pulse compression down to the bandwidth-limited pulse duration indicates a high spectral coherence. To quantify the spectral coherence, 100 output spectra $\tilde{A}(\omega)$ were simulated independently with random noise distributions and an ensemble average was calculated to obtain the firstorder coherence according to Ref. [45]. The calculations reveal a coherence of unity over the whole spectral bandwidth and, therefore, a compressibility of the output spectrum to the bandwidth-limited pulse duration.

To investigate the stability of the output pulses of the DAF with regard to the input energy, spectra and autocorrelation traces for different input pulse energies were measured. The output pulse duration decreased and the output spectral bandwidth increased with increasing input pulse energy. Changes of $\pm 5 \%$ of the input pulse energy led to less than $\pm 3 \%$ changes in the output pulse duration and less than $\pm 2 \%$ changes in the output spectral bandwidth (full width at $-30 \mathrm{~dB}$ level). The robustness against input pulse energy fluctuations increases with the number of segments of the DAF as the last segments provide only small increase in spectral bandwidth. The measurements agreed with numerical simulations with varying input energies showing the robustness against input pulse energy fluctuations. In contrast, the fiber length at which compression occurs during soliton-effect pulse compression decreases with increasing input pulse energies. This could lead to compressed output pulses which are sensitive to energy fluctuations of the input pulses or noise [37].

Another advantage of the demonstrated NLPC scheme is the robustness against the fiber length of the normally and anomalously dispersive fiber segments. Numerical simulations with different segment lengths revealed that if the pulses propagate repeatedly through normally dispersive and subsequent anomalously dispersive sections, whose lengths are not matched, only a small residual chirp results from the effective overall dispersion of the DAF. On the one hand, this chirp reduces the SPM-mediated bandwidth generation due to the reduced peak power, but can be compensated within the next fiber segments. On the other hand, a residual chirp can be beneficial in order to pre-compensate for the group velocity dispersion of optical components in the beam path, e.g., before the sample in a nonlinear microscope. For this purpose, a normally or anomalously dispersive piece of fiber can be added at the end of the DAF to achieve a residual down- or up-chirp, respectively.

To investigate the presented concept for different input pulse durations, simulations with an input energy of $1 \mathrm{~nJ}$ were performed in varying DAFs. For every simulation, the lengths of the six fiber segments were adjusted to optimize the bandwidth generation in the normally dispersive segment and the temporal recompression in the anomalously dispersive segment, respectively. A splice loss of $-0.97 \mathrm{~dB}$ was assumed for the simulations. The input pulse duration was varied between 40 and $10 \mathrm{ps}$, limited by the computational effort required for longer pulse durations. With increasing duration, the length of the first normally dispersive fiber segment could be increased on account of the weaker impact of dispersion due to the narrower bandwidth. Accordingly, the length of the first anomalously dispersive fiber segment had to be increased to compensate for the accumulated dispersion related to the acquired bandwidth. Exemplarily, the fiber length of each segment is denoted in Table 2 for an input pulse duration of $10 \mathrm{ps}$. The compressed output pulse duration is plotted as blue crosses in Fig. 5. The errors of the output pulse duration were estimated conservatively from deviations of the fiber length of each of the six fiber segments. For all input pulse durations the compressed output pulse duration was shorter than $120 \mathrm{fs}$ and decreased with reducing input pulse duration. The corresponding compression factor, shown as red circles in Fig. 5, reached a factor of about 83 for an input pulse duration of $10 \mathrm{ps,}$ however, decreased for shorter input pulses. The lower compression factors for shorter pulse durations are a result of the stronger influence of the dispersion due to the broader bandwidth. The faster temporal broadening by dispersion limits the bandwidth generation by SPM, necessary for the pulse compression. Nevertheless, due to the compensation of HOD in these DAF, the compression factors exceed (by a factor of approx. 3.5) those in a standard DAF, with which

Table 2 Length and transmission of each fiber segment of the simulated dispersion-alternating fiber chain for an input pulse duration of $10 \mathrm{ps}$

\begin{tabular}{llll}
\hline Segment & Fiber type & Length $(\mathrm{cm})$ & $\begin{array}{l}\text { Trans- } \\
\text { mission } \\
(\mathrm{dB})\end{array}$ \\
\hline 1 & DCF & 1200 & - \\
2 & SMF28 & 9700 & -0.97 \\
3 & DCF & 2 & -0.97 \\
4 & SMF28 & 14.4 & -0.97 \\
5 & DCF & 2 & -0.97 \\
6 & SMF28 & 14.4 & -0.97 \\
Total & & 10932.8 & -4.85 \\
\hline
\end{tabular}




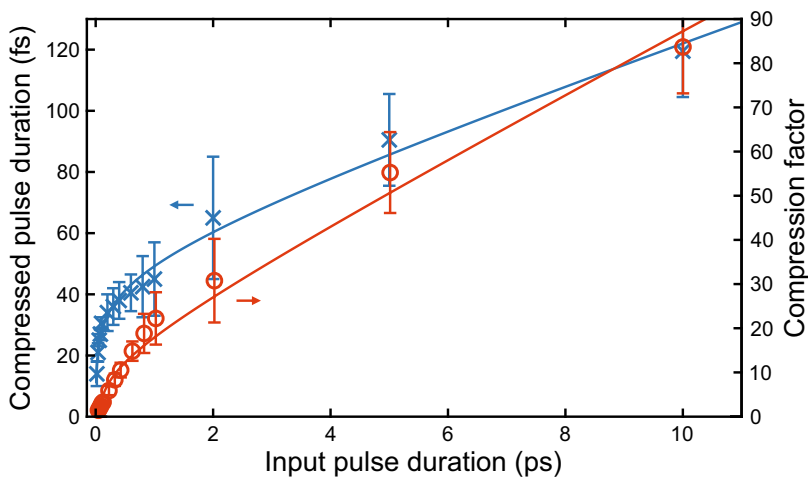

Fig. 5 Compressed output pulse duration (blue crosses) and corresponding compression factor (red circles) calculated as a function of the input pulse duration. The lines were added to guide the eye. The pulse duration errors were estimated conservatively from deviations of the fiber length of each of the six fiber segments

compression factors of 19 for $7.2 \mathrm{ps}$ input pulse duration [7] were achieved, under the limitation of HOD impact. The simulations prove that the here-presented NLPC scheme is transferable to longer input pulse durations.

\section{Conclusion}

In summary, nonlinear pulse compression in a dispersionalternating fiber with matched dispersion profiles of the involved fibers in terms of dispersion magnitude as well as slope was demonstrated. Pulse compression from $80 \mathrm{fs}$ down to the few-cycle regime with output pulse duration of 25 fs was achieved, corresponding to a compression factor of 3.2. Due to the use of a specific DCF, which matches the SMF28 dispersion in terms of magnitude and slope, the influence of HOD could be minimized, leading to bandwidth-limited pulse durations. The overall DAF transmission, which amounts to $-6.94 \mathrm{~dB}$, limited mainly from splice losses, can be improved, e.g., by an advanced fiber design or tapering to better match the mode fields of the involved fibers. Furthermore, the overall transmission can be improved by reducing the number of segments, which will consequently increase the compressed pulse duration of the output pulses. Nevertheless, a compression by a factor of 2.2 was already achieved with two segments; increasing the number of segments will then lead to higher compression factors. The robustness of the DAF concept in respect of changes or fluctuations of the input pulse energy and chirp, as well as the fiber segment lengths provides an easy-toimplement platform for nonlinear pulse compression down to the few-cycle regime. Numerical simulations predict that this concept is transferable to longer pulse durations of up to 10 ps with resulting compression factors of up to 83 .
In contrast to standard pulse compression schemes, the presented concept does not rely on high pulse energies or free-space compressors making it a versatile and simple device for many applications where all-fiber pulse compression is favorable.

Acknowledgements The authors thank Kevin W. Bennett and Corning Inc. for providing the dispersion-compensating fiber.

Funding Open Access funding enabled and organized by Projekt DEAL.

Open Access This article is licensed under a Creative Commons Attribution 4.0 International License, which permits use, sharing, adaptation, distribution and reproduction in any medium or format, as long as you give appropriate credit to the original author(s) and the source, provide a link to the Creative Commons licence, and indicate if changes were made. The images or other third party material in this article are included in the article's Creative Commons licence, unless indicated otherwise in a credit line to the material. If material is not included in the article's Creative Commons licence and your intended use is not permitted by statutory regulation or exceeds the permitted use, you will need to obtain permission directly from the copyright holder. To view a copy of this licence, visit http://creativecommons.org/licenses/by/4.0/.

\section{References}

1. I. Pupeza, S. Holzberger, T. Eidam, H. Carstens, D. Esser, J. Weitenberg, P. Rußbüldt, J. Rauschenberger, J. Limpert, T. Udem, A. Tünnermann, T.W. Hänsch, A. Apolonski, F. Krausz, E. Fill, Nat. Photonics 7, 608 (2013)

2. A. Vernaleken, J. Weitenberg, T. Sartorius, P. Rußbüldt, W. Schneider, S.L. Stebbings, M.F. Kling, P. Hommelhoff, H.-D. Hoffmann, R. Poprawe, F. Krausz, T.W. Hänsch, T. Udem, Opt. Lett. 36, 3428 (2011)

3. H. Carstens, M. Högner, T. Saule, S. Holzberger, N. Lilienfein, A. Guggenmos, C. Jocher, T. Eidam, D. Esser, V. Tosa, V. Pervak, J. Limpert, A. Tünnermann, U. Kleineberg, F. Krausz, I. Pupeza, Optica 3, 366 (2016)

4. D.R. Carlson, P. Hutchison, D.D. Hickstein, S.B. Papp, Opt. Express 27, 37374 (2019)

5. H. Zia, N.M. Lüpken, T. Hellwig, C. Fallnich, K.-J. Boller, Laser Photonics Rev. 14, 2000031 (2020)

6. H. Chen, H. Wang, M.N. Slipchenko, Y. Jung, Y. Shi, J. Zhu, K.K. Buhman, J.-X. Cheng, Opt. Express 17, 1282 (2009)

7. T. Inoue, H. Tobioka, K. Igarashi, S. Namiki, J. Lightwave Technol. 24, 2510 (2006)

8. G.T. Nogueira, B. Xu, Y. Coello, M. Dantus, F.C. Cruz, Opt. Express 16, 10033 (2008)

9. E. Sorokin, S. Naumov, I.T. Sorokina, IEEE J. Sel. Top. Quantum Electron. 11, 690 (2005)

10. C.V. Shank, R.L. Fork, R. Yen, R.H. Stolen, W.J. Tomlinson, Appl. Phys. Lett. 40, 761 (1982)

11. C. Jocher, T. Eidam, S. Hädrich, J. Limpert, A. Tünnermann, Opt. Lett. 37, 4407 (2012)

12. T. Südmeyer, F. Brunner, E. Innerhofer, R. Paschotta, K. Furusawa, J.C. Baggett, T.M. Monro, D.J. Richardson, U. Keller, Opt. Lett. 28, 1951 (2003)

13. G. McConnell, E. Riis, Appl. Phys. B 78, 557 (2004)

14. B. Schenkel, R. Paschotta, U. Keller, J. Opt. Soc. Am. B 22, 687 (2005)

15. G. Krauss, S. Lohss, T. Hanke, A. Sell, S. Eggert, R. Huber, A. Leitenstorfer, Nat. Photonics 4, 33 (2010) 
16. J. Rothhardt, S. Hädrich, H. Carstens, N. Herrick, S. Demmler, J. Limpert, A. Tünnermann, Opt. Lett. 36, 4605 (2011)

17. S. Hädrich, A. Klenke, A. Hoffmann, T. Eidam, T. Gottschall, J. Rothhardt, J. Limpert, A. Tünnermann, Opt. Lett. 38, 3866 (2013)

18. K.F. Mak, J.C. Travers, N.Y. Joly, A. Abdolvand, P. St, J. Russell, Opt. Lett. 38, 3592 (2013)

19. W. Liu, D.N. Schimpf, T. Eidam, J. Limpert, A. Tünnermann, F.X. Kärtner, G. Chang, Opt. Lett. 40, 10939 (2015)

20. Y. Liu, W. Li, D. Luo, D. Bai, C. Wang, H. Zeng, Opt. Express 24, 10939 (2016)

21. J. Schulte, T. Sartorius, J. Weitenberg, A. Vernaleken, P. Russbueldt, Opt. Lett. 41, 4511 (2016)

22. K. Kieu, R.J. Jones, N. Peyghambarian, IEEE Photonics Technol. Lett. 22, 1521 (2010)

23. A. Andrianov, A. Kim, S. Muraviov, A. Sysoliatin, Opt. Lett. 34, $3193(2009)$

24. D. Brida, G. Krauss, A. Sell, A. Leitenstorfer, Laser Photonics Rev. 8, 409 (2014)

25. J. Sotor, G. Sobon, Laser Phys. Lett. 13, 125102 (2016)

26. G. Agrawal, Nonlinear Fiber Optics (Academic Press, New York, 2013)

27. L.F. Mollenauer, R.H. Stolen, J.P. Gordon, W.J. Tomlinson, Opt. Lett. 8, 289 (1983)

28. K. Smith, L.F. Mollenauer, Opt. Lett. 14, 1284 (1989)

29. S.V. Chernikov, P.V. Mamyshev, J. Opt. Soc. Am. B 8, 1633 (1991)

30. M.A. Foster, A.L. Gaeta, Q. Cao, R. Trebino, Opt. Express 13, $6848(2005)$

31. M.V. Tognetti, H.M. Crespo, J. Opt. Soc. Am. B 24, 1410 (2007)
32. J.C. Travers, J.M. Stone, A.B. Rulkov, B.A. Cumberland, A.K. George, S.V. Popov, J.C. Knight, J.R. Taylor, Opt. Express 15, 13203 (2007)

33. M.L.V. Tse, P. Horak, J.H.V. Price, F. Poletti, F. He, D.J. Richardson, Opt. Lett. 31, 3504 (2006)

34. G. Fan, T. Balčiūnas, C. Fourcade-Dutin, S. Haessler, A.A. Voronin, A.M. Zheltikov, F. Gérôme, F. Benabid, A. Baltuška, T. Witting, Opt. Express 24, 12713 (2016)

35. P. Colman, C. Husko, S. Combrié, I. Sagnes, C.W. Wong, A. De Rossi, Nat. Photonics 4, 862 (2010)

36. E.A. Anashkina, A.V. Andrianov, S.V. Muravyev, A.V. Kim, Opt. Express 19, 20141 (2011)

37. S. Boscolo, C. Finot, Shaping Light in Nonlinear Optical Fibers (Wiley, Chichester, 2017)

38. A.A. Voronin, A.M. Zheltikov, Phys. Rev. A 78, 063834 (2008)

39. M.D. Pelusi, H.F. Liu, IEEE J. Quantum Electron. 33, 1430 (1997)

40. T. Inoue, S. Namiki, Laser Photonics Rev. 2, 83 (2008)

41. S.K. Turitsyn, B.G. Bale, M.P. Fedoruk, Phys. Rep. 521, 135 (2012)

42. J.M. Dudley, S. Coen, G. Genty, Rev. Mod. Phys. 78, 1135 (2006)

43. J.Y. Lee, D.Y. Kim, Opt. Express 14, 11608 (2006)

44. B. Yang, J. Duan, Z. Xie, H. Xiao, J. Cent. South Univ. 20, 1832 (2013)

45. J.M. Dudley, S. Coen, IEEE J. Sel. Top. Quantum Electron. 8, 651 (2002)

Publisher's Note Springer Nature remains neutral with regard to jurisdictional claims in published maps and institutional affiliations. 